\title{
Pharmacogenetics of Anticoagulation and Clinical Events in Warfarin-Treated Patients: A Register-Based Cohort Study with Biobank Data and National Health Registries in Finland
}

This article was published in the following Dove Press journal:

Clinical Epidemiology

Anna-Leena Vuorinen (D)
Mika Lehto iD
Mikko Niemi
Kari Harno
Juha Pajula
Mark van Gils
Jaakko Lähteenmäki

'VTT Technical Research Centre of
Finland, Tampere, Finland; ${ }^{2}$ Heart and
Lung Center, Helsinki University
Hospital, Helsinki, Finland; ${ }^{3}$ University of
Helsinki, Helsinki, Finland; ${ }^{4}$ Department
of Clinical Pharmacology and
Individualized Drug Therapy Research
Program, University of Helsinki, Helsinki,
Finland; ${ }^{5}$ Department of Clinical
Pharmacology, HUS Diagnostic Center,
Helsinki University Hospital, Helsinki,
Finland; ${ }^{6}$ Department of Health and
Social Management, University of Eastern
Finland, Kuopio, Finland; ${ }^{7}$ VTT Technical
Research Centre of Finland, Espoo,
Finland

Finland
Purpose: To assess the association between VKORC1 and CYP2C9 variants and the incidence of adverse drug reactions in warfarin-treated patients in a real-world setting.

Materials and Methods: This was a register-based cohort study (PreMed) linking data from Finnish biobanks, national health registries and patient records between January 1st 2007 and June 30th 2018. The inclusion criteria were: 1) $\geq 18$ years of age, 2) CYP2C9 and VKORC1 genotype information available, 3) a diagnosis of a cardiovascular disease, 4) at least one warfarin purchase, 5) regular INR tests. Eligible individuals were divided into two warfarin sensitivity groups; normal responders, and sensitive and highly sensitive responders based on their VKORC1 and CYP2C9 genotypes. The incidences of clinical events were compared between the groups using Cox regression models.

Results: The cohort consisted of 2508 participants (45\% women, mean age of 69 years), of whom $65 \%$ were categorized as normal responders and $35 \%$ sensitive or highly sensitive responders. Compared to normal responders, sensitive and highly sensitive responders had fewer INR tests below 2 (median: 33.3\% vs 43.8\%, 95\% CI: $-13.3 \%$, $-10.0 \%$ ) and more above 3 (median: $18.2 \%$ vs $6.7 \%, 95 \% \mathrm{Cl}: 8.3 \%, 10.8 \%$ ). The incidence (per 100 patient-years) of bleeding outcomes was 5.4 for normal responders and 5.6 for the sensitive and highly sensitive responder group (HR=1.03, 95\% CI: 0.74, 1.44). The incidence of thromboembolic outcomes was 4.9 and 7.8 , respectively $(\mathrm{HR}=1.48,95 \% \mathrm{CI}: 1.08,2.03)$.

Conclusion: In a real-world setting, genetically sensitive and highly sensitive responders to warfarin had more high INR tests and required a lower daily dose of warfarin than normal responders. However, the risk for bleeding events was not increased in sensitive and highly sensitive responders. Interestingly, the risk of thromboembolic outcomes was lower in normal responders compared to the sensitive and highly sensitive responders.

Trial Registration: NCT04001166.

Keywords: pharmacogenomics, warfarin, bleeding, INR, CYP2C9, VKORC1

\section{Introduction}

For decades warfarin has been a commonly used oral anticoagulant for the treatment and prevention of thromboembolic events. ${ }^{1,2}$ Although efficacious, warfarin treatment is challenged by a narrow therapeutic range and high inter-individual variation in treatment response which increases the risk of treatment complications.
Correspondence: Anna-Leena Vuorinen VTT Technical Research Centre of Finland, P.O. Box 1300, Tampere, 33101, Finland

Tel +358408485966

Email anna-leena.vuorinen@vtt.fi 
Warfarin is a leading cause of severe adverse drug reactions and is responsible for up to one-third of adverse drug reaction-related emergency hospitalizations and fatalities. $^{3-5}$

Approximately $50-60 \%$ of the inter-individual variability in the warfarin dose requirement is explained by genetic factors, particularly with variants in CYP2C9 and $V K O R C 1{ }^{6-8}$ Individuals carrying decreased function alleles of $C Y P 2 C 9$ (eg, $C Y P 2 C 9 * 2$ and $C Y P 2 C 9 * 3$ ) have a reduced ability to metabolize warfarin, and those carrying decreased function variants of $V K O R C 1$ are more sensitive to warfarin. ${ }^{6}$ Thus, they require lower warfarin doses to achieve therapeutic anticoagulation measured as an international normalized ratio of the prothrombin time (INR) in the range of $2-3 .{ }^{10}$ Individuals with these variant alleles are more likely to experience overanticoagulation which might increase the risk for bleeding complications. $^{11-13}$

A number of pharmacogenetic studies have assessed the effect of genotype-guided warfarin dosing, however, the evidence on its benefits remains contradictory. ${ }^{6,14-18}$ Studies conducted are criticized for their small sample sizes and limited follow-up times, and for the use of surrogate end-points (ie, anticoagulation related parameters) rather than clinical events such as bleeding or thrombosis. ${ }^{19-21}$ Few studies have investigated the impact of the variant alleles on the incidence of clinical events, and most of them have been unpowered to show significant differences. It has been shown that the incidence of hemorrhagic events is higher among genetically sensitive individuals with the risk for major hemorrhages increasing up to 2-5 fold. ${ }^{12,13,20,22,23}$ However, not all the studies have confirmed these associations. ${ }^{24-26}$ Moreover, it seems that the risk of minor bleeding events is not increased in variant allele carriers. ${ }^{23,27}$ The association between genotype and the risk of clinical events remains therefore unclear.

The large genome data resources of rapidly evolving biobanks linked with clinical data provide opportunities for pharmacogenetic research. Although causal inference is limited with the use of such resources, they provide a unique possibility to overcome some methodological challenges including insufficient sample sizes and the use of clinical outcome events. The aim of the current study was to assess the association between VKORC1 and $C Y P 2 C 9$ variants and the incidence of adverse drug reactions in warfarin-treated patients in a real-world setting by using a register-linkage design with multiple Finnish biobanks and national health registries.

\section{Materials and Methods Study Design and Data Sources}

This was a register-based cohort study (PreMed study) linking data from multiple Finnish biobanks and national health registries to investigate pharmacogenetics of antithrombotic drugs. Three biobanks (THL Biobank, Auria Biobank, Helsinki Biobank) first identified individuals genotyped in biobank studies for the selected genetic variants and with an eligible disease diagnosis (see Participants). These data together with the patients' demographic data were then linked with individual's medication purchases retrieved from the drug reimbursement registry maintained by the Social Insurance Institution of Finland (Kela) to form the original PreMed cohort. The registry contains complete information on all reimbursed drugs dispensed from Finnish pharmacies with the following information: the date of purchase, identification code (ATC code) of the medication and the purchased amount in milligrams and in defined daily doses (DDD). The cohort data were further linked to laboratory data from the registries of hospital districts and municipalities, and to health care encounter data that included diagnosis codes of outpatient visits and inpatient care using nationwide registers, the Care Register for Health Care and the Register of Primary Health Care Visits, both maintained by the Finnish Institute for Health and Welfare. The two registers contain data from all specialized and primary care episodes, respectively. The data were retrieved for the period of January 1st 2007-June 30th 2018, and the participants were followed until December 31st 2018. Data linking was done using the personal identity code assigned to every individual residing in Finland.

\section{Participants}

Individuals fulfilling the following inclusion criteria were included in the study: 1$) \geq 18$ years of age at the first date of warfarin purchase, 2) CYP2C9 (*2 and *3 alleles; rs1799853 and rs1057910) and VKORC1 g.3588G $>\mathrm{A}$ (rs9923231) genotype information available in the biobank, 3) a diagnosis of a cardiovascular disease (Supplementary material, Table S1), 4) at least one warfarin purchase between January 1st 2007 and June 30th $2018,5)$ at least three INR test results. Individuals who 
had made warfarin purchases between January 1st 2005 and December 31st 2006 were excluded to ensure a washout period of two years.

\section{Genotyping}

Genotyping of the participants had been carried out in the framework of the on-going FinnGen project ${ }^{28}$ and past research projects of the Finnish Institute for Health and Welfare. ${ }^{29,30}$ The observed genotype frequencies were similar to those previously observed in the European population, and the three variants were in Hardy-Weinberg equilibrium (Unpublished data). On the basis of the VKORC1 and CYP2C9 genotypes, patients were divided into three warfarin responder type groups; normal responders, sensitive responders, and highly sensitive responders. $^{12}$

\section{Warfarin Exposure}

Warfarin exposure was defined as a period of a patient's continuous warfarin purchases and INR tests. We first identified the date of the first warfarin purchase for each participant and set this as an initial start date for warfarin exposure. Then, INR data of each patient were analysed. If there were INR test results taken up to 30 days before the first warfarin purchase, the start date of warfarin exposure was corrected with the first date of these INR measurements. Warfarin exposure between consecutive purchases was deemed continuous until the earliest of the following: 1) the time difference between two INR test results was more than 60 days, 2) the time difference between two consecutive warfarin purchases was more than 500 days and all the purchased drugs had been consumed (based on the DDD information), 3) a direct oral anticoagulant (DOAC) or a heparin product was purchased (Supplementary material, Table S1), 4) the study followup ended (December 31st 2018).

\section{Variables \\ Bleeding Events}

The primary outcome was the occurrence of a bleeding event that occurred during warfarin exposure and was associated with one of the defined diagnosis codes (Supplementary material, Table S1). Recurrent bleeding events, referring to cases where the patient was diagnosed with a bleeding event before warfarin exposure and again during the exposure, were defined as outcomes, only if they were combined with a reduction in hemoglobin levels of $20 \mathrm{~g} / \mathrm{dl}$, or greater. The same hemoglobin reduction degree was used for identifying major bleeding events. For all clinical outcome events, the participants were followed from the first day of the warfarin exposure to the occurrence of the outcome event, or to the last date of exposure, whichever came first. In addition, only the first event occurring after the exposure and the corresponding follow-up was analyzed.

\section{Thromboembolic Events}

Clinical events occurring during the exposure and associated with one of the defined diagnosis codes (Supplementary material, Table S1) were defined as thromboembolic outcomes. If a thromboembolic event with the identical ICD-10 code had occurred before the warfarin exposure, the subsequent thromboembolic event was deemed as an outcome only if combined with a hospital admission. Moreover, we used a blanking period of seven days indicating that the recurrent thromboembolic events occurring within seven days from the beginning of the exposure were excluded to ensure that the event was actually a new event, and not incorrectly interpreted as an outcome due to a delayed diagnosis registration.

\section{Bleeding-related Hospitalizations}

Hospital admissions (admission date, discharge date and related ICD-10 diagnosis code) were extracted from the Care Register for Health Care. All bleeding-related hospitalizations in specialized care occurring during the warfarin exposure were included in the analyses.

\section{INR Data}

INR tests taken during the exposure time were extracted from the laboratory data and analyzed in detail to investigate the anticoagulation effect. Restricting the INR data to the first 90 days, we calculated the following parameters: the number of INR tests, the time taken to reach the therapeutic range in days, the time in the therapeutic range (TTR) with interpolation according to the Rosendaal method, ${ }^{31}$ and the occurrence of below- and above-range INR levels (defined as INR $<2.0$ and INR $>3.0$ ). The desired therapeutic range for INR is between 2 and 3 for most indications. ${ }^{10}$ The minimum number of INR test results per patient was three, otherwise the parameters were defined as missing values.

\section{Drug Dosage}

Average daily doses were calculated by dividing the total purchased amount of warfarin by the length of the exposure period. 


\section{Covariates}

Information on sex and age was obtained via biobanks. The indication for warfarin use was identified on the basis of ICD-10 codes using the inclusion diagnosis that occurred closest before the warfarin initiation. Kidney function was investigated from laboratory data by applying the glomerular filtration rate (GFR) ${ }^{32}$

Significant interaction drugs (Supplementary material, Table S1) were identified based on class $\mathrm{C}$ and class $\mathrm{D}$ clinical significance using the inxbase drug-drug interaction database (https://www.medbase.fi/en/professionals/ inxbase). The patient was considered exposed to an interacting drug if the drug was purchased at least once during warfarin exposure. The interacting drugs were categorized in two groups: drugs that increase the risk of bleeding events, and drugs that increase the risk for thromboembolic events. Acetylsalicylic acid (ASA) was included in the list of bleeding risk-increasing drugs. The information on ASA use was obtained from medical records as ASA is an over-the-counter drug and thus those purchases are not included in the drug reimbursement registry.

\section{Statistical Analyses}

The initial inquiries in the biobanks indicated that 2678 patients with genotype data could be recruited from the three biobanks. Assuming a risk of bleeding complications of $5 \%^{33}$ and the prevalence of CYP2C9 variant allele carriers of $35 \%,{ }^{34}$ setting an alpha level of 0.05 and the power of 0.9 , the estimated number of participants enabled us to detect an increase in the bleeding risk from $5 \%$ to $9 \%$.

Baseline characteristics were presented for all study participants and across the three responder type groups: normal responders, sensitive responders and highly sensitive responders. Similarly, the INR-related parameters were calculated across the responder type groups. We used nonparametric confidence intervals and estimators for the difference in the location parameters to assess the differences in the INR parameters. To investigate the number of patients who achieved therapeutic range, risk ratio (RR) and its 95\% confidence interval (CI) were calculated.

The occurrence of outcome events was presented in terms of the incidence rates (per 100 patient years) for each responder type group. We used Cox proportional hazards regression models to investigate the associations between the responder type group and the occurrence of outcome events and bleeding-related hospitalizations. The follow-up time was restricted to 730 days in the main analyses. Both crude and adjusted results on hazard ratios (HR) and the 95\% CIs were computed. The adjusted analyses included sex, age at the beginning of warfarin exposure, a diagnosis of cancer (during the warfarin exposure or up to 2 years before the warfarin use), and the indication for warfarin use as potential confounders. In addition, the adjusted models for bleeding-related outcomes included the use of bleeding risk-increasing drugs as a covariate, and, correspondingly, the thromboembolicrelated outcomes included the use of thromboembolic riskincreasing drugs as a covariate.

We also performed three sensitivity analyses. First, we analyzed the occurrences of outcome events using the whole follow-up time instead of restricting it to 730 days, and then further decreasing the follow-up to 90 days. Secondly, we excluded the recurrent events from the analyses to include first-time diagnoses only. In the third analysis, an INR value of 3 or higher was required to coexist together with a bleeding event.

All analyses were conducted in $\mathrm{R}$ Studio version 1.1.456 using $\mathrm{R}$ version 3.5.1 (2018-07-02).

\section{Ethical Aspects}

This study was conducted in accordance with the Declaration of Helsinki. The study was approved by the ethics committee of the Hospital District of Helsinki and Uusimaa (HUS/513/2019), and by all involved register controllers. After the data linkage in the biobanks, all the data were pseudonymized. The informed consent of the patients was obtained through the biobanks as defined in the Finnish Biobank Act 2013 (688/2012). ${ }^{35}$ The study was registered in the clinicaltrials.gov (NCT04001166).

\section{Results}

The original PreMed cohort consisted of 7005 patients. Of those, $36 \%(n=2508)$ fulfilled the eligibility criteria for the current study by having made at least one warfarin purchase and having at least three INR test results (Figure 1).

Table 1 shows the characteristics of the study participants. Of the 2508 participants, 1133 (45.2\%) were women and the mean age was 68.8 years. Based on the different variant alleles of the 2508 patients, 1627 (65\%) were categorized as normal responders, $802(32 \%)$ as sensitive responders, and $79(3 \%)$ as highly sensitive responders. The most common inclusion diagnoses were atrial fibrillation and vascular disease (coronary artery or peripheric 


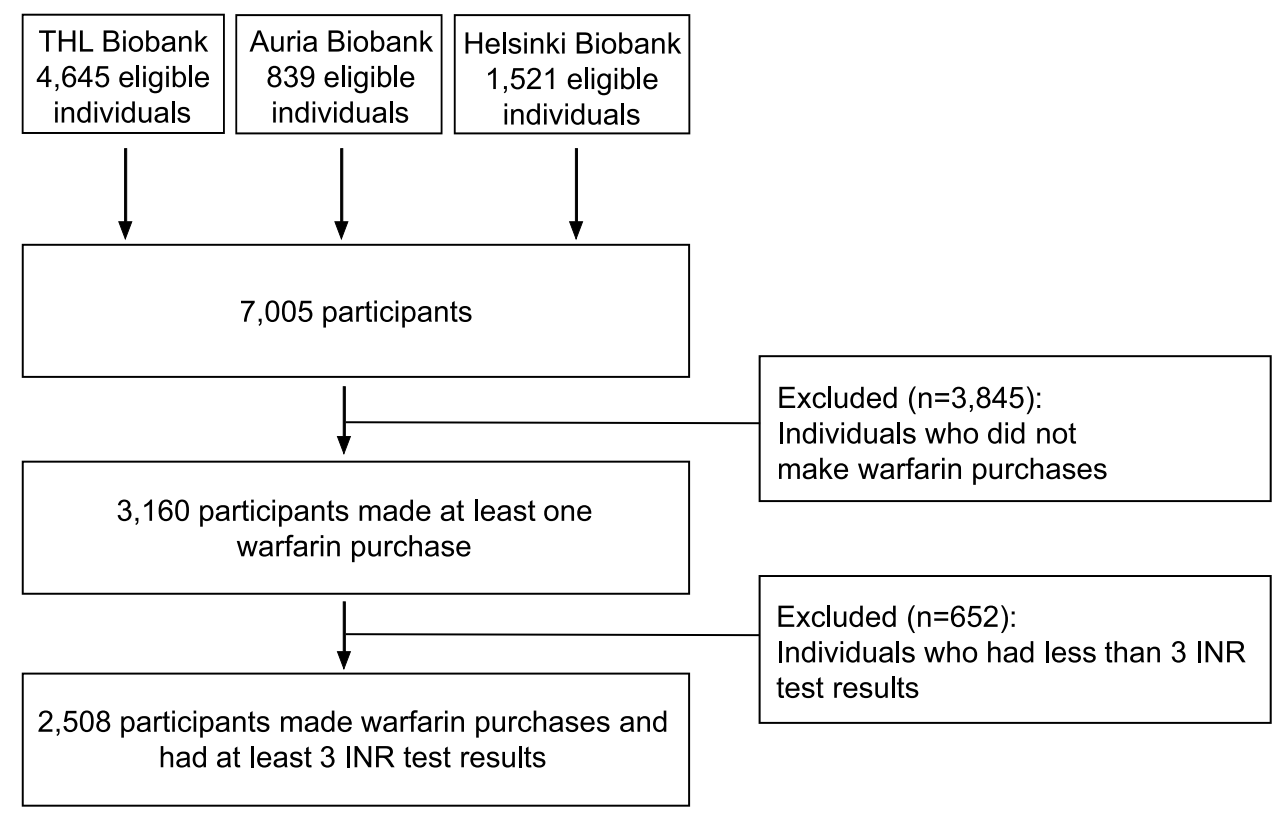

Figure I Participant recruitment and eligibility.

Abbreviation: INR, international normalized ratio.

artery disease). The sociodemographic characteristics and inclusion diagnoses did not differ across the responder type groups. A cancer was diagnosed more frequently in normal and sensitive responders than in highly sensitive responders. The exposure time was longer in normal responders than in sensitive and highly sensitive responders (372, 333 and 254 days, respectively). Moreover, the percentage of patients who changed from warfarin to DOACs differed between the groups with a decreasing trend toward highly sensitive responders $(6.8 \%, 4.6 \%$ and $1.3 \%$ ). There were no differences in the use of interacting drugs between the genotype groups. The numbers (\%) of ASA users were 269 (17\%), 122 (15\%), 13 (17\%) for normal responders, sensitive responders, and highly sensitive responders. The mean daily dose of warfarin was lower among highly sensitive responders than in sensitive and normal responders $(2.6 \mathrm{mg} / \mathrm{d}$ vs $4.0 \mathrm{mg} / \mathrm{d}$ vs and $5.5 \mathrm{mg} / \mathrm{d}$, respectively).

Table 2 shows the INR parameters for the responder type groups during the first 90 days. The median number of INR tests was 12. The median percentage of the time in the therapeutic range and the time to reach the therapeutic range did not differ between the groups. Compared to normal responders, the sensitive and highly sensitive responders had fewer INR test results below $2(33.3 \%$ vs $43.8 \%$, 95\% CI for median difference: $-13.3 \%,-10.0 \%)$ and more above $3(18.2 \%$ vs
$6.7 \%, 95 \%$ CI for median difference: $8.3 \%, 10.8 \%$ ) during the first 90 days on warfarin.

During the median follow-up of 318 days, 149 bleeding events occurred, resulting in 5.5 events per 100 patient-years (Table 3). There were 97 and 52 bleeding events in the normal responder and sensitive responder groups, respectively. No events occurred in the highly sensitive group. Therefore, all subsequent outcome event analyses were performed by combining the sensitive and highly sensitive groups. The risk of bleeding events did not differ between the two responder type groups (HR=1.03, 95\% CI: 0.74, 1.44), (Table 3 and Figure 2 ). There was no difference in the bleeding-related hospitalizations $(\mathrm{HR}=0.89,95 \% \mathrm{CI}: 0.51,1.55)$. However, the incidence of thromboembolic events was higher in the sensitive and highly sensitive group with 4.9 and 7.8 events per 100 patient-years in the normal, and sensitive and highly sensitive responder groups, respectively. Compared to normal responders, the risk was 1.48 -fold for the sensitive and highly sensitive group (95\% CI: $1.08,2.03$ ) (Figure 3). Adjusting for potential confounders decreased the risk estimate for thromboembolic events to $1.37(95 \%$ CI: $1.00,1.88)$. The variantspecific HRs for bleeding events are presented in the Supplementary material (Table S2); the risk for bleeding events was increased in individuals who were homozygous for $C Y P 2 C C^{*} 2$ compared to non-carriers of the 
Table I Characteristics of the Study Participants

\begin{tabular}{|c|c|c|c|c|}
\hline & $\begin{array}{c}\text { All Study } \\
\text { Participants }\end{array}$ & $\begin{array}{c}\text { Normal } \\
\text { Responders }\end{array}$ & $\begin{array}{c}\text { Sensitive } \\
\text { Responders }\end{array}$ & $\begin{array}{c}\text { Highly Sensitive } \\
\text { Responders }\end{array}$ \\
\hline $\begin{array}{l}\text { Number of participants } \\
\text { Sex, female, n (\%) } \\
\text { Age, years, mean (sd) }\end{array}$ & $\begin{array}{c}2508 \\
1133(45.2) \\
68.8(10.4)\end{array}$ & $\begin{array}{c}1627 \\
726(44.6) \\
68.8(10.4)\end{array}$ & $\begin{array}{c}802 \\
374(46.6) \\
68.9(10.5)\end{array}$ & $\begin{array}{c}79 \\
33(4 I .8) \\
67.9(9.1)\end{array}$ \\
\hline $\begin{array}{l}\text { Indication for warfarin use } \\
\text { Atrial fibrillation, } \mathrm{n}(\%) \\
\text { Vascular disease, } \mathrm{n}(\%) \\
\text { Pulmonary embolism, } \mathrm{n}(\%) \\
\text { Stroke or atherosclerosis in cerebral } \\
\text { arteries, } \mathrm{n}(\%) \\
\text { Phlebitis or thrombophlebitis, } \mathrm{n}(\%) \\
\text { Other venous thrombosis, } \mathrm{n}(\%)\end{array}$ & $\begin{array}{l}1596(63.6) \\
352(14.0) \\
199(8.0) \\
127(5.1) \\
181(7.2) \\
53(2.1)\end{array}$ & $\begin{array}{l}1028(63.2) \\
235(14.4) \\
122(7.5) \\
78(4.8) \\
124(7.6) \\
40(2.5)\end{array}$ & $\begin{array}{l}511(63.7) \\
107(13.4) \\
74(9.2) \\
45(5.6) \\
52(6.5) \\
13(1.6)\end{array}$ & $\begin{array}{c}57(72.1) \\
10(12.7) \\
3(3.8) \\
4(5.1) \\
5(6.3) \\
0\end{array}$ \\
\hline $\begin{array}{l}\text { VKORCI } \\
\text { GG, n (\%) } \\
\text { AG, n (\%) } \\
\text { AA, n (\%) }\end{array}$ & $\begin{array}{l}96 \mid(38.3) \\
I 183(47.2) \\
364(14.5)\end{array}$ & & & \\
\hline $\begin{array}{l}\text { CYP2C9 } \\
\text { CYP2C9*I } 1 * 1, \mathrm{n}(\%) \\
\text { CYP2C9*1/*2, n (\%) } \\
\text { CYP2C9*1/*3, n (\%) } \\
\text { CYP2C9*2/*2, n (\%) } \\
\text { CYP2C9*2/*3, n (\%) } \\
\text { CYP2C9*3/*3, n (\%) }\end{array}$ & $\begin{array}{c}1690(67.4) \\
473(18.9) \\
274(10.9) \\
24(1.0) \\
36(1.4) \\
11(0.4)\end{array}$ & & & \\
\hline $\begin{array}{l}\text { Exposure time, days, median [IQR] } \\
\text { Changed to DOAC, } n(\%) \\
\text { Changed to heparin products, n (\%) }\end{array}$ & $\begin{array}{c}352[123-1200] \\
148(5.9) \\
413(16.5)\end{array}$ & $\begin{array}{c}372[137-1256] \\
110(6.8) \\
277(17.0)\end{array}$ & $\begin{array}{c}333[116-1110] \\
37(4.6) \\
127(15.8)\end{array}$ & $\begin{array}{c}254[96-843] \\
\text { I (I.3) } \\
9(11.4)\end{array}$ \\
\hline $\begin{array}{l}\text { Interacting drugs } \\
\text { Increasing risk of bleeding events, n (\%) } \\
\text { Increasing risk of thromboembolic events, } \\
\mathrm{n}(\%)\end{array}$ & $\begin{array}{c}1833(73.1) \\
16(0.6)\end{array}$ & $\begin{array}{c}1195(73.5) \\
\quad I 1(0.7)\end{array}$ & $\begin{array}{c}581(72.4) \\
5(0.6)\end{array}$ & $\begin{array}{c}57(72.2) \\
0\end{array}$ \\
\hline $\begin{array}{l}\text { Kidney function, GFR, mean (sd) } \\
\text { Diagnosis of cancer }{ }^{\mathrm{a}}, \mathrm{n}(\%) \\
\text { Genotyped in the clinic }{ }^{\mathrm{b}}, \mathrm{n}(\%) \\
\text { Daily dose, } m g / d, \text { median }[\mathrm{IQR}]\end{array}$ & $\begin{array}{c}79.1(19.0) \\
\text { missing=325 } \\
492(19.6) \\
7(0.3) \\
5.0[3.7-6.7]\end{array}$ & $\begin{array}{c}79.3(19.2) \\
\text { missing=21 I } \\
333(20.5) \\
5(0.3) \\
5.5[4.3-7.1]\end{array}$ & $\begin{array}{c}79.0(18.8) \\
\text { missing=99 } \\
\text { I50 (I8.7) } \\
\text { I }(0.1) \\
4.0[3.0-5.3]\end{array}$ & $\begin{array}{c}77.6(18.7) \\
\text { missing=I5 } \\
9(11.4) \\
\text { I }(1.3) \\
2.6[1.9-5.0]\end{array}$ \\
\hline
\end{tabular}

Notes: ${ }^{\mathrm{a} C a n c e r}$ diagnosed during the warfarin exposure or up to 2 years before the warfarin initiation; ${ }^{\mathrm{b}} \mathrm{A}$ pharmacogenetic test for $\mathrm{CYP} 2 \mathrm{C} 9$ and $\mathrm{VKORCI}$ performed to guide warfarin dosing.

Abbreviations: DOAC, direct oral anticoagulant; GFR, glomerular filtration rate; IQR, interquartile range; SD, standard deviation.

allele $(\mathrm{HR}=3.35,95 \% \mathrm{CI}: 1.24,9.09)$. There were no significant differences in the bleeding risk in relation to the VKORC1 or CYP2C $9 * 3$ allele carrier status (CYP2C $9 * 3$ : HR $=0.74,95 \%$ CI: $0.42,1.27$; VKORC1: GG vs $\mathrm{AG} \mathrm{HR}=0.87,95 \%$ CI: $0.61,1.23$; GG vs AA $\mathrm{HR}=0.88$ 95\% CI: $0.53,1.46$ ).

The sensitivity analyses showed that changing the length of the follow-up time either by analyzing the whole follow-up or by restricting it to the first 90 days did not alter the estimates (Table 4). Excluding recurrent thromboembolic events from the analyses strengthened the association between the genotype groups and the risk of thromboembolic events by increasing the HR to 1.82 (95\% CI: $1.23,2.71)$ in sensitive and highly sensitive responders. There were no recurrent bleeding events and thus those analyses were not repeated. 


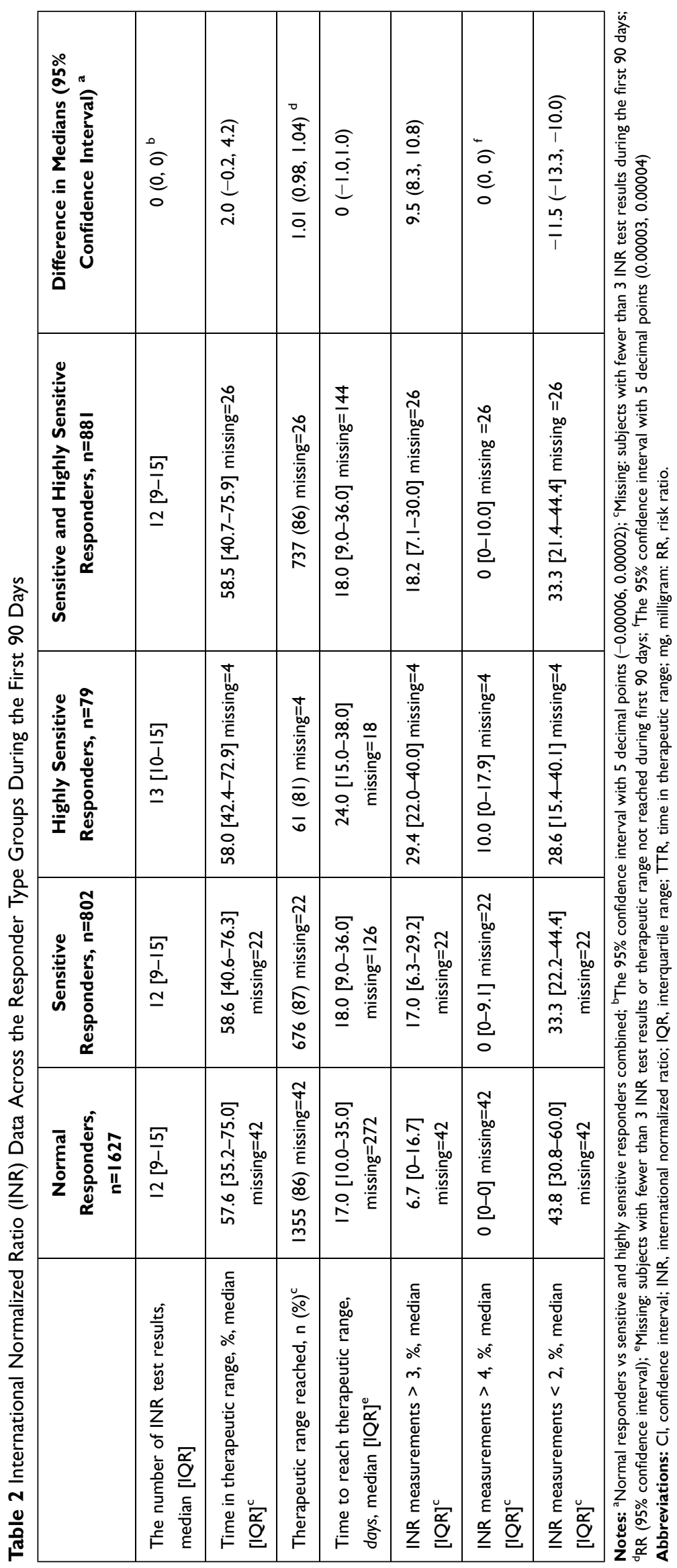




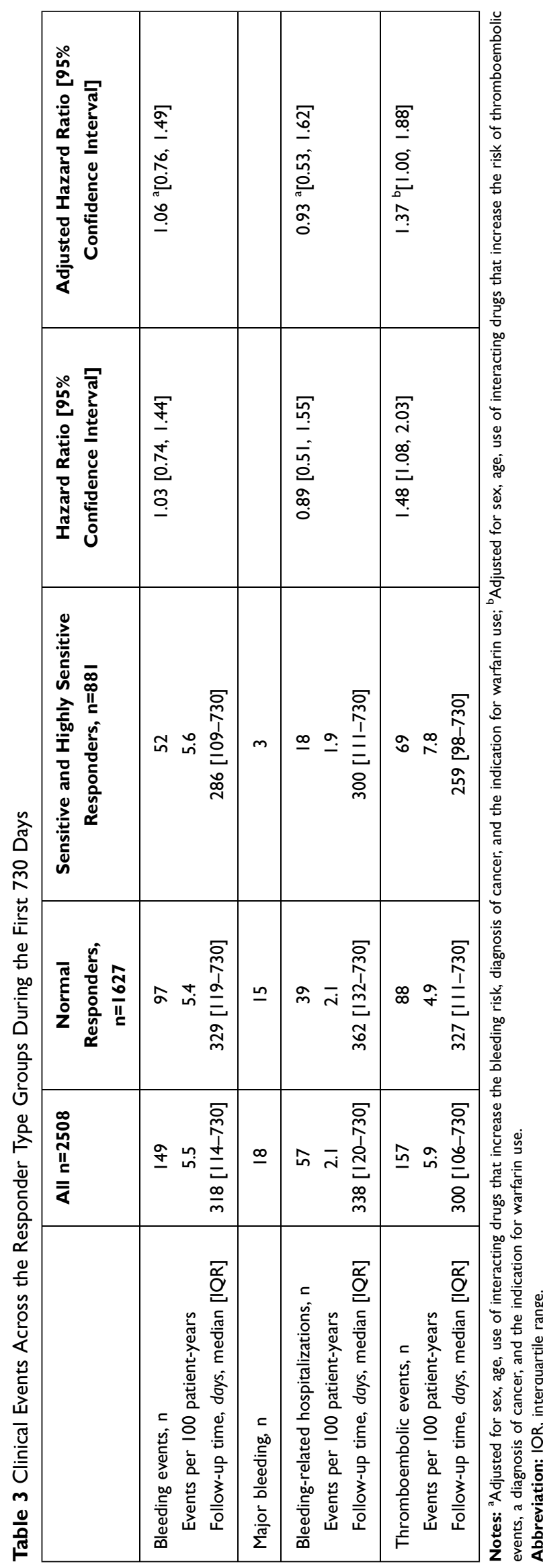

\section{Discussion}

The results of this study showed that in a real-world setting, the genetically sensitive and highly sensitive responders to warfarin did not have an increased risk of bleeding complications when compared to normal responders. Nevertheless, they had more out-of-range INR test results and required a lower daily dose of warfarin than normal responders. In addition, we found that sensitive and highly sensitive responders to warfarin had a higher risk of thromboembolic events. This was one of the largest studies investigating the impact of genetic variants on the efficacy of warfarin and clinical outcome events.

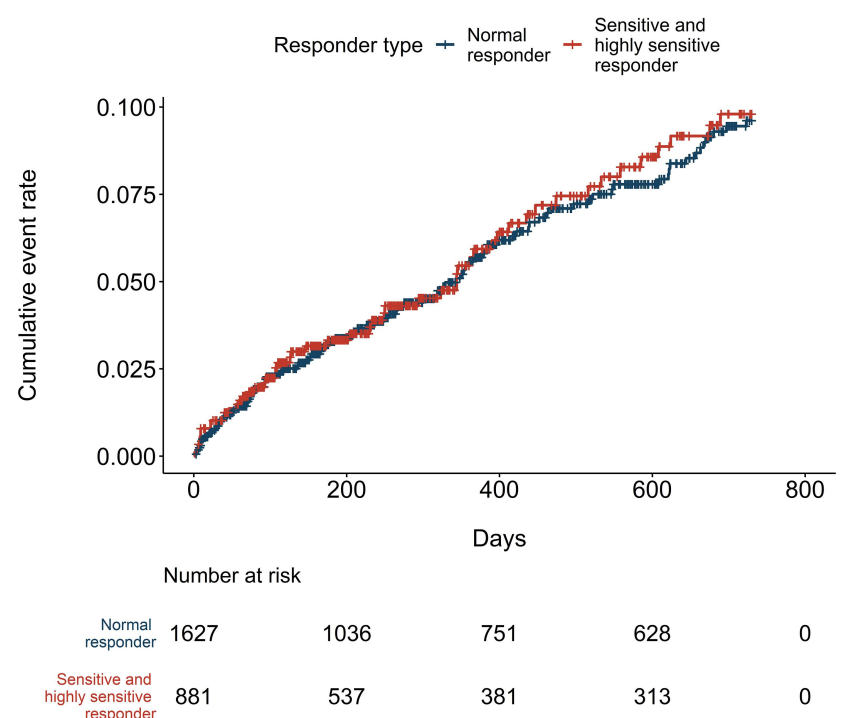

Figure 2 Cumulative incidence of bleeding events.

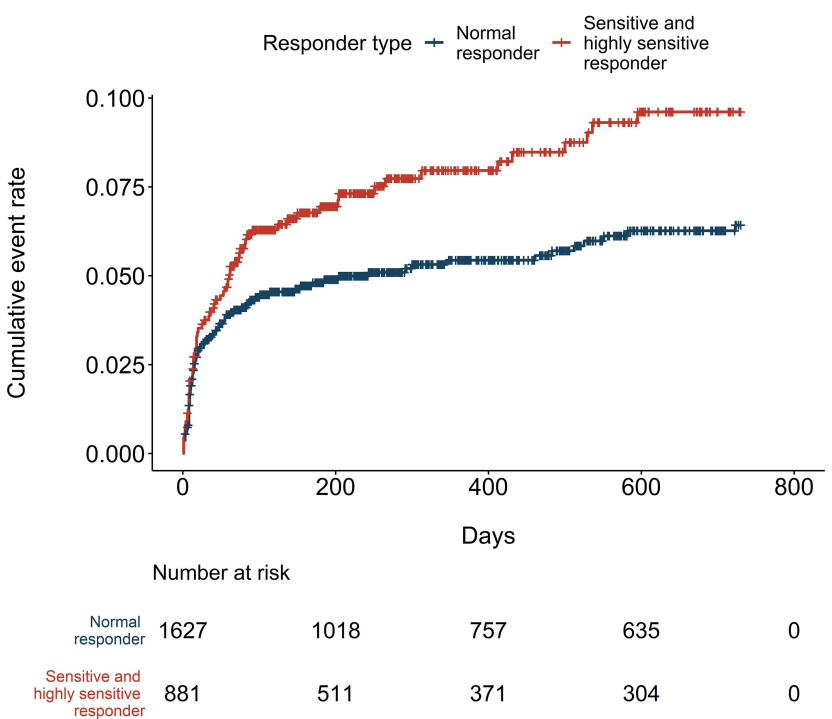

Figure 3 Cumulative incidence of thromboembolic events. 


\begin{tabular}{|c|c|c|}
\hline 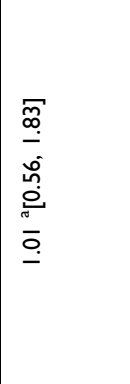 & 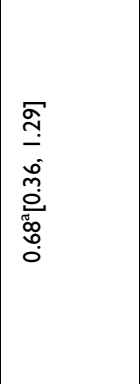 & 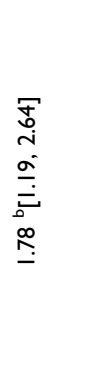 \\
\hline 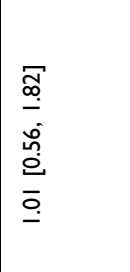 & 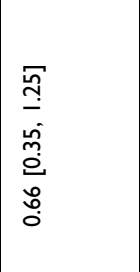 & 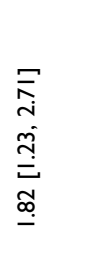 \\
\hline 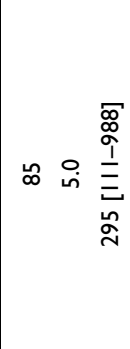 & 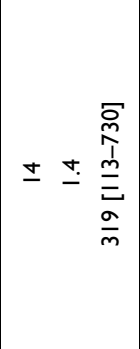 & 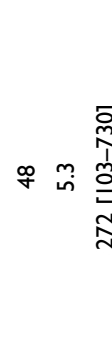 \\
\hline 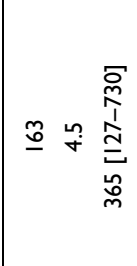 & 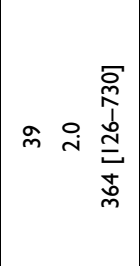 & 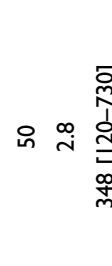 \\
\hline 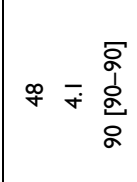 & 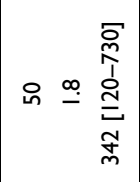 & 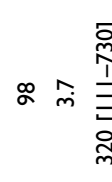 \\
\hline 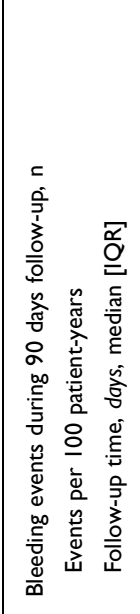 & 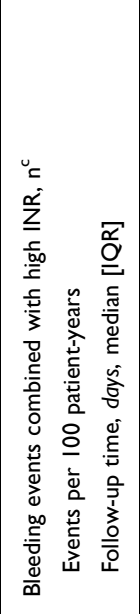 & 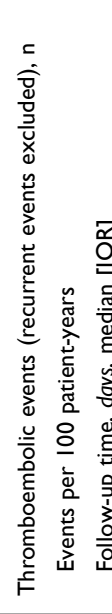 \\
\hline
\end{tabular}

We found that among all study participants the median percentage of time in the therapeutic range (TTR) was $58 \%$ during the first 90 days, and the TTR was not dependent on the genotype. It has been suggested that sufficient anticoagulation is achieved with a minimum threshold for a TTR ranging from 58\%-65\%, whereas patients with a TTR $\leq 40 \%$ have shown a notable risk of clinical complications. ${ }^{33,36}$ The outcome was optimal in those patients with a TTR higher than $80 \% .^{33}$ This indicates that the majority of participants in this study achieved sufficient anticoagulation despite their genetic susceptibility to over-anticoagulation, which might further relate to our neutral findings on the bleeding risk. The TTR level seems to have been in line with other registry-based studies $^{36}$ although it was slightly lower than in the Finnish nationwide FinWAF-study. ${ }^{33}$ However, the mean TTR in the FinWAF, $62 \%$ was achieved with the mean follow-up time of 3.2 years, while in the present study the TTR was analyzed for only the first 90 days on warfarin including the high variation period.

Although sensitive and highly sensitive responders had more INR test results above 3 , the risk of bleeding complications, regardless of the follow-up time used in the analyses, was not higher when compared to normal responders. Neither was there a difference in bleedingrelated hospitalizations. In line with our findings, several studies have reported an increased anticoagulation effect and lower dose requirements to be associated with these genetic alleles. ${ }^{6,12,20,37-39}$ Some studies have shown an increased bleeding risk with $C Y P 2 C 9$ and $V K O R C 1$ allele variants, ${ }^{11-13,39}$ while in most studies as well as in the meta-analysis of Jorgensen et al the allele variants have not been associated with increased risk of bleeding. ${ }^{24,40-42}$ On the other hand, in the very large Phase III trials, HOKUSAI-VTE and ENGAGE AF-TIMI 48 (together more than 8000 patients on warfarin), genetic polymorphisms in CYP2C9 and VKORC1 affected the safety of warfarin. ${ }^{11,12}$ However, this finding was underscored in patients with highly sensitive genotype, whilst the number of those was only modest in our study. Park et al suggested that clinical events are more associated with patients' characteristics and clinical practices than with genetic factors. ${ }^{42}$ Our findings support this interpretation; sensitive and highly sensitive responders had lower daily doses of warfarin indicating that the over-anticoagulation was detected and corrected with dose adjustment. Another factor contributing to the neutral bleeding risk could be that we included all bleeding events, whereas the increased 
risk is frequently seen concerning major bleeding events only. In fact, Tomek et al showed that minor bleeding events alone were not associated with genotype. ${ }^{27}$

The sensitivity analyses showed that the incidence of bleeding events did not differ between the responder type groups regardless of whether we investigated the risk either during the first 90 days or over the whole followup of approximately 318 days. Some studies have suggested that the bleeding risk is higher during warfarin initiation in particular. ${ }^{12,20,41}$

The increased risk of thromboembolic events among sensitive and highly sensitive responders remains unclear, and contradictory to our INR test result findings. Compared to normal responders, sensitive and highly sensitive responders had nearly a 1.5 -fold risk of suffering thromboembolic events, which further increased almost 2-fold when recurrent thromboembolic events were excluded despite that these individuals had significantly fewer INR test results below 2. However, the INR test results showed that sensitive responders and highly sensitive responders, in particular, had more INR tests above 3. Individuals with high INR values are sometimes advised to pause warfarin treatment or adjust warfarin dose, which might actually expose them to thromboembolic outcomes. Moreover, a lower percentage of highly sensitive responders reached the therapeutic range during the first 90 days, and they reached it seven days later on average. It seems that highly sensitive responders had greater challenges finding the optimal warfarin dose.

\section{Limitations}

The findings of this study should be interpreted in the light of the following limitations. First, although data obtained from nationwide registries have been validated and have been shown to be of good quality, ${ }^{43,44}$ in this study the use of registry data posed limitations to investigating warfarin exposure and the identification of outcomes. Estimating warfarin exposure through drug purchase data did not reveal whether and when the warfarin was actually used. In an attempt to increase the likelihood of true warfarin exposure, we required that the patient adhered to regular INR tests according to national guidelines in Finland.We consider the use of INR data together with warfarin purchases as a strength of this study which is not frequently used in other register-based studies. In terms of identifying thromboembolic outcomes, we cannot rule out the possibility of misclassification of the patients' prior events as an outcome event. To minimize such bias, events with the same diagnosis codes were included only if they occurred at least seven days after the first diagnosis and were combined with a hospital admission. In addition, we conducted sensitivity analyses in which we excluded all recurrent events, and those results consistently showed a risk-increasing effect. For bleeding outcomes, bleeding-related hospital admissions were separately analyzed, and those results also indicated a small risk of misclassification.

Second, despite the study including 2508 participants, the sample size was not sufficient to investigate differences across the three genotype groups. No bleeding events occurred among highly sensitive responders, and therefore, we needed to combine sensitive and highly sensitive responder groups. Interestingly, the results of the INR data indicated that the highly sensitive responders faced most challenges with anticoagulation suggesting an increased risk of bleeding events. Moreover, although the sample size remained slightly lower than we anticipated, this study found a significant association between $C Y P 2 C 9 * 2$ and a bleeding risk (HR of 3.35), which was used as a basis for sample size calculations, and which was also in line with earlier reported studies. ${ }^{20,23,46}$

Third, we had no data on mortality. Thus, we may have ignored cases of fatal bleedings where the patient died without being admitted to hospital. However, we did not expect death rates to differ between the genotypes as the variants investigated in this study are frequently related to non-fatal clinical outcomes.

Fourth, there may still be residual bias present in the study as we were able to control only a few possible confounding factors. We did not have data on behavioral factors such as smoking, alcohol use and dietary factors, vitamin $\mathrm{K}$ intake in particular, that influence anticoagulation and hence they could explain the results. In addition, information on the body size affecting dose requirements was not available. ${ }^{47}$

\section{Conclusions}

In a real-world setting, the genetically sensitive and highly sensitive responders to warfarin had more INR test results above 3 and required a lower daily dose of warfarin than normal responders. However, the risk of bleeding events was not significantly increased for the sensitive and highly sensitive responders. On the contrary, the risk of thromboembolic outcomes was lower for the normal responders. Our results suggest that 
genotype-guided warfarin dosing may have limited advantages in preventing bleeding complications but might help to identify patients who are at a high risk of thromboembolic events.

\section{Abbreviations}

ASA, acetylsalicylic acid; CI, confidence interval, DDD, defined daily doses; DOAC, direct oral anticoagulant; GFR, glomerular filtration rate; HR, hazard ratio; INR, international normalized ratio; IQR, interquartile range; $\mathrm{mg}$, milligram; $\mathrm{RR}$ risk ratio; $\mathrm{SD}$, standard deviation; TTR, time in therapeutic range.

\section{Data Sharing Statement}

No further data will be shared. The data permits granted for the study by the biobanks and the national health registries do not allow sharing of individual-level data outside the research group.

\section{Acknowledgments}

The data used for the research were obtained from Auria Biobank (study number: AB19-9833), Helsinki Biobank (study number: HBP20190038) and THL Biobank (study number: BB2019_6). We thank all the study participants for their generous participation in the biobank research. We would also like to thank Merja Perälä, Perttu Terho and Mikko Tukiainen from the Auria Biobank, Theresa Knopp, Miika Koskinen and Otto Manninen from the Helsinki Biobank and Niina Eklund, Anni Joensuu and Katariina Peltonen from the THL Biobank for their contribution in defining the subcohorts for the study.

This study was funded by the alliance between Business Finland and VTT Technical Research Centre of Finland, and the following sponsors: Oy Karl Fazer AB, Novartis Finland Oy, Pfizer Oy, Roche Diagnostics Oy, Avaintec Oy, Crown CRO Oy, Mediconsult Oy, Biobank Cooperative Finland. The study sponsors had no involvement in the data collection, analysis, or interpretation of the data; the writing of the article; or the decision to submit the article for publication. A conference presentation that was based on the findings reported in this article has been published previously in abstract form in the Virtual Annual Meeting \& Poster Session of the Pharmacogenomics Research Network (PGRN), October 26th 2020.

\section{Author Contributions}

All authors made substantial contributions to conception and design, acquisition of data, or analysis and interpretation of data; took part in drafting the article or revising it critically for important intellectual content; agreed to submit to the current journal; gave final approval of the version to be published; and agree to be accountable for all aspects of the work.

\section{Disclosure}

Dr Juha Pajula, Dr Mark Van Gils, and Mr Jaakko Lahteenmaki reports grant from Business Finland, grants from Oy Karl Fazer AB, grants from Novartis Finland Oy, grants from Pfizer Oy, grants from Roche Diagnostics Oy, grants from Avaintec Oy, grants from Crown CRO Oy, grants from Mediconsult Oy, grants from Biobank Cooperative Finland, outside the submitted work. The authors declare that they have no other competing interests in this work.

\section{References}

1. Johnson JA, Gong L, Whirl-Carrillo M, et al. Clinical pharmacogenetics implementation consortium guidelines for CYP2C9 and VKORC1 genotypes and warfarin dosing. Clin Pharmacol Ther. 2011;90(4):625-629. doi:10.1038/clpt.2011.185

2. Yang J, Chen Y, Li X, et al. Influence of CYP2C9 and VKORC1 genotypes on the risk of hemorrhagic complications in warfarin-treated patients: a systematic review and meta-analysis. Int J Cardiol. 2013;168(4):4234-4243. doi:10.1016/j.ijcard.2013.07. 151

3. Budnitz DS, Lovegrove MC, Shehab N, Richards CL. Emergency hospitalizations for adverse drug events in older Americans. $N$ Engl $J$ Med. 2011;365(21):2002-2012. doi:10.1056/NEJMsa1103053

4. Shehab N, Lovegrove MC, Geller AI, Rose KO, Weidle NJ, Budnitz DS. US Emergency department visits for outpatient adverse drug events, 2013-2014. JAMA. 2016;316(20):2115. doi:10.1001/ jama.2016.16201

5. Lapatto-Reiniluoto O, Patinen L, Niemi M, Backman JT, Neuvonen PJ. Drug-related inadvertent deaths in a university hospital - a declining trend. Basic Clin Pharmacol Toxicol. 2015;117 (6):421-426. doi:10.1111/bcpt.12435

6. Johnson JA, Cavallari LH. Warfarin pharmacogenetics. Trends Cardiovasc Med. 2015;25(1):33-41. doi:10.1016/j.tcm.2014.09.001

7. Johnson JA, Caudle KE, Gong L, et al. Clinical Pharmacogenetics Implementation Consortium (CPIC) Guideline for pharmacogenetics-guided warfarin dosing: 2017 Update. Clin Pharmacol Ther. 2017;102(3):397-404. doi:10.1002/cpt.668

8. Wadelius M, Chen LY, Lindh JD, et al. The largest prospective warfarin-treated cohort supports genetic forecasting. Blood. 2009;113(4):784-792. doi:10.1182/blood-2008-04-149070

10. Hindrics G, Potpara T, Dagres N, et al. ESC Guidelines for the diagnosis and management of atrial fibrillation developed in collaboration with the European Association of Cardio-Thoracic Surgery (EACTS). Eur Heart J. 2020. doi:10.1093/eurheartj/ehaa612

11. Vandell AG, Walker J, Brown KS, et al. Genetics and clinical response to warfarin and edoxaban in patients with venous thromboembolism. Heart. 2017;103(22):1800-1805. doi:10.1136/ heartjnl-2016-310901 
12. Mega JL, Walker JR, Ruff CT, et al. Genetics and the clinical response to warfarin and edoxaban: findings from the randomised, double-blind ENGAGE AF-TIMI 48 trial. Lancet. 2015;385 (9984):2280-2287. doi:10.1016/S0140-6736(14)61994-2

13. Aithal GP, Day CP, Kesteven PJ, Daly AK. Association of polymorphisms in the cytochrome P450 CYP2C9 with warfarin dose requirement and risk of bleeding complications. Lancet. 1999;353 (9154):717-719. doi:10.1016/S0140-6736(98)04474-2

14. Pirmohamed M, Burnside G, Eriksson N, et al. A randomized trial of genotype-guided dosing of warfarin. $N$ Engl J Med. 2013;369 (24):2294-2303. doi:10.1056/NEJMoa1311386

15. Kimmel SE, French B, Kasner SE, et al. A pharmacogenetic versus a clinical algorithm for warfarin dosing. $N$ Engl J Med. 2013;369 (24):2283-2293. doi:10.1056/NEJMoa1310669

16. Jorgensen AL, FitzGerald RJ, Oyee J, Pirmohamed M, Williamson PR. Influence of CYP2C9 and VKORC1 on patient response to warfarin: a systematic review and meta-analysis. Novelli Ged. PLoS One. 2012;7(8):e44064. doi:10.1371/journal. pone. 0044064

17. Epstein RS, Moyer TP, Aubert RE, et al. Warfarin genotyping reduces hospitalization rates. $J$ Am Coll Cardiol. 2010;55 (25):2804-2812. doi:10.1016/j.jacc.2010.03.009

18. Gage BF, Bass AR, Lin H, et al. Effect of genotype-guided warfarin dosing on clinical events and anticoagulation control among patients undergoing hip or knee arthroplasty: the GIFT Randomized clinical trial. JAMA. 2017;318(12):1115-1124. doi:10.1001/jama.2017.11469

19. Carnes CA. What is the role of pharmacogenetics in optimization of warfarin dosing? Trends Cardiovasc Med. 2015;25(1):42-43. doi:10.1016/J.TCM.2014.10.003

20. Higashi MK, Veenstra DL, Kondo LM, et al. Association between CYP2C9 genetic variants and anticoagulation-related outcomes during warfarin therapy. JAMA. 2002;287(13):1690. doi:10.1001/jama. 287.13.1690

21. Ruff CT. Pharmacogenetics of Warfarin Therapy. Clin Chem. 2018;64(11):1558-1559. doi:10.1373/clinchem.2017.284927

22. Yang T, Zhou Y, Chen C, Lu M, Ma L, Cui Y. Genotype-guided dosing versus conventional dosing of warfarin: a meta-analysis of 15 randomized controlled trials. J Clin Pharm Ther. 2019;44 (2):197-208. doi:10.1111/jcpt.12782

23. Limdi N, McGwin G, Goldstein J, et al. Influence of CYP2C9 and VKORC1 $1173 \mathrm{C} / \mathrm{T}$ genotype on the risk of hemorrhagic complications in african-american and european-american patients on warfarin. Clin Pharmacol Ther. 2008;83(2):312. doi:10.1038/SJ.CLPT.6100290

24. Swanson KM, Zhu Y, Rojas RL, et al. Comparing outcomes and costs among warfarin-sensitive patients versus warfarin-insensitive patients using the right drug, right dose, right time: using genomic data to individualize treatment (RIGHT) $10 \mathrm{~K}$ warfarin cohort. PLoS One. 2020;15:5. doi:10.1371/JOURNAL.PONE.0233316

25. Ogg MS, Brennan P, Meade T, Humphries SE. CYP2C9*3 allelic variant and bleeding complications. Lancet. 1999;354(9184):1124. doi:10.1016/S0140-6736(05)76918-X

26. Schwarz UI, Ritchie MD, Bradford Y, et al. Genetic determinants of response to warfarin during initial anticoagulation. $N$ Engl $J$ Med. 2008;358(10):999-1008. doi:10.1056/NEJMoa0708078

27. Tomek A, Mat’oška $\mathrm{V}$, Kolářová $\mathrm{T}$, et al. The bleeding risk during warfarin therapy is associated with the number of variant alleles of CYP2C9 and VKORC1 genes. Cardiology. 2013;125:3. doi:10.1159/ 000350407

28. FinnGen research project. https://www.finngen.fi/en. Accessed September 19, 2020.

29. Borodulin K, Vartiainen E, Peltonen M, et al. Forty-year trends in cardiovascular risk factors in Finland. Eur J Public Health. 2015;25 (3):539-546. doi:10.1093/eurpub/cku174
30. Aromaa A, Koskinen S. Health and Functional Capacity in Finland: Baseline Results of the Health 2000 Health Examination Survey. Helsinki: KTL, National Public Health Institute Finland; 2004. https://www.julkari.fi/handle/10024/78534.

31. Rosendaal F, Cannegieter S, van der Meer F, Briët E. A method to determine the optimal intensity of oral anticoagulant therapy. Thromb Haemost. 1993;69(3):236-239. doi:10.1055/s-0038-1651587

32. Levey AS, Stevens LA, Schmid CH, et al. A new equation to estimate glomerular filtration rate. Ann Intern Med. 2009;150 (9):604-612. doi:10.7326/0003-4819-150-9-200905050-00006

33. Lehto M, Niiranen J, Korhonen P, et al. Quality of warfarin therapy and risk of stroke, bleeding, and mortality among patients with atrial fibrillation: results from the nationwide FinWAF registry. Pharmacoepidemiol Drug Saf. 2017;26(6):657-665. doi:10.1002/ pds. 4194

34. Sistonen J, Fuselli S, Palo JU, Chauhan N, Padh H, Sajantila A. Pharmacogenetic variation at CYP2C9, CYP2C19, and CYP2D6 at global and microgeographic scales. Pharmacogenet Genomics. 2009;19(2):170-179. doi:10.1097/FPC.0b013e32831ebb30

35. Finnish biobank Act (688/2012). http://www.finlex.fi/fi/laki/alkup/ 2012/20120688\#Lid2180503. Accessed September 19, 2020.

36. Gateman D, Trojnar ME, Agarwal G. Time in therapeutic range: warfarin anticoagulation for atrial fibrillation in a community-based practice. Can Fam Physician. 2017;63:e425-e431.

37. Wadelius M, Sörlin K, Wallerman $\mathrm{O}$, et al. Warfarin sensitivity related to CYP2C9, CYP3A5, ABCB1 (MDR1) and other factors. Pharmacogenomics J. 2004;4(1):40-48. doi:10.1038/sj.tpj.6500220

38. Biss TT, Avery PJ, Williams MD, Brandão LR, Grainger JD. The VKORC1 and CYP2C9 genotypes are associated with over-anticoagulation during initiation of warfarin therapy in children. J Thromb Haemost. 2013;11(2):373-375. doi:10.1111/jth. 12072

39. Baker WL, Johnson SG. Pharmacogenetics and oral antithrombotic drugs. Curr Opin Pharmacol. 2016;27:38-42. doi:10.1016/J.COPH. 2016.01.008

40. Jorgensen AL, FitzGerald RJ, Oyee J, Pirmohamed M, Williamson PR. Influence of CYP2C9 and VKORC1 on patient response to warfarin: a systematic review and meta-analysis. PLoS One. 2012;7:8. doi:10.1371/journal.pone.0044064

41. Roth JA, Boudreau D, Fujii MM, et al. Genetic risk factors for major bleeding in patients treated with warfarin in a community setting. Clin Pharmacol Ther. 2014;95(6):636-643. doi:10.1038/clpt.2014.26

42. Park YK, Lee MJ, Kim JH, et al. Genetic and non-genetic factors affecting the quality of anticoagulation control and vascular events in atrial fibrillation. J Stroke Cerebrovasc Dis. 2017;26(6):1383-1390. doi:10.1016/j.jstrokecerebrovasdis.2017.02.022

43. Furu K, Wettermark B, Andersen M, Martikainen JE, Almarsdottir AB, Sørensen HT. The nordic countries as a cohort for pharmacoepidemiological research. Basic Clin Pharmacol Toxicol. 2010;106(2):86-94. doi:10.1111/j.1742-7843.2009.00494.x

44. Rapola JM, Virtamo J, Korhonen P, et al. Validity of diagnoses of major coronary events in national registers of hospital diagnoses and deaths in Finland. Eur J Epidemiol. 1997;13(2):133-138. doi:10.10 23/A:1007380408729

46. Meckley LM. An analysis of the relative effects of VKORC1 and CYP2C9 variants on anticoagulation related outcomes in warfarin-treated patients. Thromb Haemost. 2008;100(2):229-239. doi:10.1160/TH07-09-0552

47. Jonas DE, McLeod HL. Genetic and clinical factors relating to warfarin dosing. Trends Pharmacol Sci. 2009;30(7):375-386. doi:10.1016/j.tips.2009.05.001 


\section{Publish your work in this journal}

Clinical Epidemiology is an international, peer-reviewed, open access, online journal focusing on disease and drug epidemiology, identification of risk factors and screening procedures to develop optimal preventative initiatives and programs. Specific topics include: diagnosis, prognosis, treatment, screening, prevention, risk factor modification,

Submit your manuscript here: https://www.dovepress.com/clinical-epidemiology-journal systematic reviews, risk \& safety of medical interventions, epidemiology \& biostatistical methods, and evaluation of guidelines, translational medicine, health policies \& economic evaluations. The manuscript management system is completely online and includes a very quick and fair peer-review system, which is all easy to use. 